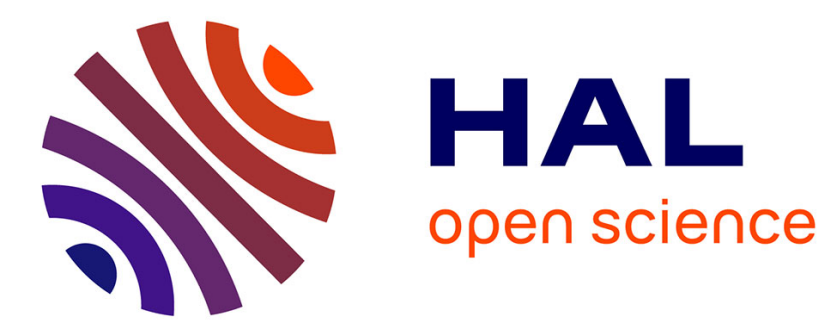

\title{
L'émergence de négociations collectives autonomes en Chine
}

Chloé Froissart

\section{To cite this version:}

Chloé Froissart. L'émergence de négociations collectives autonomes en Chine. Critique Internationale, 2014. hal-03119518

\section{HAL Id: hal-03119518 https://hal.science/hal-03119518}

Submitted on 13 Mar 2021

HAL is a multi-disciplinary open access archive for the deposit and dissemination of scientific research documents, whether they are published or not. The documents may come from teaching and research institutions in France or abroad, or from public or private research centers.
L'archive ouverte pluridisciplinaire HAL, est destinée au dépôt et à la diffusion de documents scientifiques de niveau recherche, publiés ou non, émanant des établissements d'enseignement et de recherche français ou étrangers, des laboratoires publics ou privés. 


\section{Critique \\ internationale \\ Revue comparative de sciences sociales}

\section{Les conflits du travail dans le monde}

L'échec de l'institutionnalisation des conflits du travail dans les mines d'Afrique du Sud par Raphaël Botiveau

Crise économique et contestation sociale en Espagne : des syndicats percutés par les mouvements sociaux?

par Sophie Béroud

L'émergence de négociations collectives autonomes en Chine par Chloé Froissart

Les conflits du travail en Allemagne : nouvelles formes, nouveaux enjeux par Olivier Giraud et Michel Lallement

Le mouvement immigré du printemps 2006 : vers un retour des mobilisations politiques de travailleurs aux États-Unis? par Mathieu Bonzom 
Numéro 65 - octobre-décembre 2014 Trimestriel

\section{Critique internationale}

Revue comparative de sciences sociales 


\section{L'émergence de négociations collectives autonomes en Chine}

par Chloé Froissart

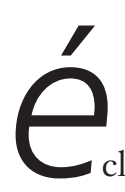

clairant un phénomène nouveau négligé par les chercheurs, qui en dénient parfois l'existence et même la possibilité ${ }^{1}$, cet article cherche à démontrer qu'il existe bien des négociations collectives autonomes en Chine, au sens où elles sont initiées et menées par des travailleurs organisés - même si ceuxci ont parfois besoin de la caution de l'État ou du Parti pour négocier - selon un protocole qu'ils tentent de faire respecter par les employeurs, les autorités locales et les syndicats officiels. Tout en nous intéressant aux pratiques de la négociation, nous chercherons également à expliquer comment de telles négociations sont possibles dans un système politique qui ne reconnaît ni le droit de grève ni le droit d'association. Notre argument principal est que l'apolitisme affiché par les acteurs leur permet, au nom de considérations rationnelles et pragmatiques que le Parti peut difficilement désavouer, de faire progressivement évoluer les modes de représentation et de gestion des conflits, et que les modes d'exercice du pouvoir du régime autoritaire chinois s'en trouvent par là même renégociés.

\footnotetext{
1. Fang Lee Cooke, « New Dynamics of Industrial Conflicts in China: Causes, Expressions and Resolution Alternatives », dans Gregor Gall (ed.), New Forms and Expressions of Conflict at Work, New York, Palgrave Macmillan, 2013, p. 108-129; Chris King-Chi Chan, Elaine Sio-leng Hui, « The Development of Collective Bargaining in China: From "Collective Bargaining by Riot" to "Party State-led Wage Bargaining" », The China Quarterly, 217, mars 2014, p. 221-242.
} 
Nous nous appuierons pour ce faire sur l'analyse de 30 conflits qui, à l'exception de deux, se sont déroulés dans la province du Guangdong au Sud de la Chine entre septembre 2010 et le printemps 2014. Ces conflits sont étudiés à partir de différentes sources : des rapports internes rédigés par quatre $\mathrm{ONG}^{2}$ et un cabinet d'avocats qui soutiennent les négociations ${ }^{3}$, tous situés dans le Guangdong (à l'exception d'une organisation basée à Hong Kong qui conseille les autres ${ }^{4}$ ) ; des entretiens approfondis, conduits en avril 2014 auprès de plusieurs membres de ces organisations et du cabinet d'avocats ainsi qu'auprès de cinq représentants ouvriers issus de différentes entreprises ; enfin l'observation, en septembre 2013, de réunions organisées dans les locaux des ONG pour élaborer les stratégies de négociation. Tous les types d'entreprises sont représentés dans cet échantillon qui compte 8 entreprises hongkongaises, 6 entreprises chinoises privées, 4 entreprises d'État, 3 entreprises japonaises dont une multinationale, 4 coréennes, 3 taïwanaises, une américaine et une entreprise à capitaux mixtes, en majorité des PME et des entreprises de taille intermédiaire. Leur point commun est que la majorité de leurs employés sont des travailleurs d'origine rurale ou, dans le cas des entreprises d'État, que les conflits concernent ces travailleurs migrants.

\section{Un cadre institutionnel insatisfaisant}

\section{Des droits individuels sans droits collectifs}

Bien que la Chine n'ait cessé d'évoluer vers une économie capitaliste depuis la réintroduction de l'économie de marché en 1978, le système institutionnel et idéologique qui régit les relations professionnelles demeure profondément ancré dans son passé communiste. Si les droits individuels des ouvriers et des employés ont beaucoup progressé ces dernières années, les droits collectifs ne sont toujours pas reconnus, ce qui rend très aléatoire la garantie des droits individuels ${ }^{5}$.

En complément de la Loi sur le travail de 1994, une Loi sur les contrats de travail publiée en 2007 a rendu obligatoire la signature d'un contrat pour tous les travailleurs et toutes les formes d'emploi. Cette mesure, qui constitue une avancée importante dans la formalisation des relations salariales, renforce la protection des employés, régularise l'emploi temporaire et favorise l'emploi de longue durée tout en instaurant un système d’ancienneté. La nouvelle loi sur la sécurité sociale

\footnotetext{
2. Bien que ce label soit contestable en Chine où il n'existe pas de société civile institutionnalisée, nous l'employons à la fois par commodité et parce que les organisations que nous avons étudiées revendiquent leur autonomie face à l'État et se désignent elles-mêmes comme des ONG.

3. Ces rapports, qui nous ont été transmis par les organisations pour les besoins de notre recherche et dans l'espoir que nous puissions faire connaître leur action, ne sont cependant pas publics. Aussi n'y ferons-nous pas directement référence.

4. Les modalités de ce partenariat ne seront pas développées pour préserver la sécurité de nos interlocuteurs. 5. Feng Chen, « Individual Rights and Collective Rights: Labor's Predicament in China », Communist and PostCommunist Studies, 40, 2007, p. 59-79.
} 
de 2011, quant à elle, instaure un système de protection sociale pour tous les travailleurs (y compris les migrants venus de la campagne) qui inclut une assurance vieillesse, maladie, chômage, accident du travail et maternité, ainsi qu'un fonds pour l'accès au logement.

Il n'existe cependant pas de liberté syndicale ni de droit de grève. La Fédération nationale des syndicats chinois (FNSC), organisation de masse créée par le Parti et qui lui est toujours entièrement subordonnée, réunit tous les syndicats. Organisés hiérarchiquement (au niveau de l'usine, puis du district urbain, de la municipalité, de la province, et au niveau national), ceux-ci sont responsables devant les échelons supérieurs de leur hiérarchie, non devant les employés. Si la Loi sur les syndicats (1992, révisée en 2001) leur donne le droit de se former au niveau des usines, c'est à la condition qu'ils obtiennent l'approbation des échelons supérieurs de la FNSC et qu'ils reconnaissent son rôle dirigeant. Aussi toute tentative de fonder un syndicat indépendant a-t-elle toujours été sévèrement réprimée. Comme le note Feng Chen, « le fait que toute forme d'organisation indépendante reste illégale reflète l'intention du Parti de prévenir toute alternative collective au sein de la société susceptible d'offrir une ouverture de choix politique à des individus isolés $»^{6}$.

Les syndicats souffrent par ailleurs d'une incapacité structurelle à représenter les travailleurs. Ils jouent traditionnellement le rôle de « courroie de transmission » du Parti dans les usines, supervisant le processus de production et organisant des services sociaux pour la classe ouvrière. Selon la loi de 2001, ils sont censés représenter à la fois les intérêts des employés, ceux des employeurs et ceux de la nation tout entière, l'idée implicite étant que tous ces intérêts sont identiques, ou du moins qu'ils ne sont pas antagonistes. En fait, leur double mission est contradictoire puisque cette loi dispose que leur rôle est tout autant de protéger les droits des ouvriers que d'« aider les entreprises à augmenter la productivité et à améliorer l'efficacité économique ». Le droit de grève n'étant pas reconnu ${ }^{7}$, les syndicats sont tenus de prévenir les arrêts de travail, sinon de favoriser la reprise de la production (art. 27). En cas de conflit, ils se considèrent donc - au mieux - comme des médiateurs entre employeurs et employés. Toutefois, dans la mesure où ils sont la plupart du temps nommés par la direction, qui les rémunère et dont ils sont souvent également membres, les représentants syndicaux au sein des entreprises se rangent du côté des employeurs.

6. Ibid., p. 8. Les citations sont traduites par nos soins.

7. Inscrite dans les Constitutions de 1975 et de 1978, la « liberté de grève » a été supprimée de la Constitution de 1982, toujours en vigueur. La Chine n'a ratifié que quatre des huit conventions fondamentales de l'OIT et toujours pas celle relative au droit de grève et à la liberté syndicale. La grève n'est cependant pas formellement interdite dans la législation chinoise, ce qui permet dans la pratique une gestion plus souple de cette dernière par les autorités. 


\section{Des contrats collectifs sans négociations collectives}

A priori, rien ne semble donc plus éloigné de la conception chinoise officielle des relations professionnelles que la notion de négociation collective entendue comme un processus auquel employeurs et employés prennent part sur un pied d'égalité, fondé sur le droit d'organisation autonome des travailleurs et sur le droit de grève - permettant de contrebalancer une relation conçue comme essentiellement conflictuelle et déséquilibrée - afin d'établir des contrats collectifs améliorant les conditions de travail.

Parce qu'il implique la reconnaissance du caractère conflictuel des relations professionnelles, le terme de «négociations collectives » (jiti tanpan) est très peu utilisé par les autorités chinoises, qui lui préfèrent le terme de « consultations collectives » (jiti xieshang). La législation qui prévoit la tenue de telles consultations au sein des entreprises en vue de l'établissement de contrats collectifs n'a cessé d'être étoffée depuis plus de quinze ans. Cependant, de nombreux observateurs, chinois comme étrangers, soulignent que ces contrats ne sont qu'une réplique des conditions minimum légales et que l'augmentation importante de leur nombre est avant tout la conséquence d'une compétition bureaucratique pour atteindre des objectifs chiffrés. D’une manière générale, les employés n'étant la plupart du temps même pas consultés, le processus achoppe sur leur manque de participation effective aux négociations et sur l'incapacité structurelle des syndicats à les représenter ${ }^{8}$.

\section{L'évolution des conflits}

Ces dernières années, les grèves n'ont cessé d'augmenter en nombre et en radicalité9. Alors qu'ils s'étaient longtemps contentés de demander à leurs employeurs l'application du salaire minimum, les travailleurs réclament désormais un partage plus juste des profits au sein de l'entreprise ainsi que la garantie de droits sociaux. Ils remettent clairement en cause l'arbitraire patronal et syndical et demandent parfois une refonte des syndicats.

8. Feng Chen, « Individual Rights and Collective Rights: Labor's Predicament in China »; Simon Clarke, Chang Hee Lee, Qi Li, « Collective Consultation and Industrial Relations in China », British fournal of Industrial Relations, 42 (2), 2004, p. 235-254, C. King-Chi Chan, E. Sio-leng Hui, « The Development of Collective Bargaining in China: From "Collective Bargaining by Riot" to "Party State-led Wage Bargaining" », art. cité, p. 226-227.

9. Dans la mesure où le droit de grève n'est pas reconnu en Chine, il n'existe pas de statistiques officielles dans ce domaine. Seules sont disponibles les statistiques concernant les conflits traités par médiation ou par arbitrage ou portés devant les tribunaux. Tous les observateurs s'accordent cependant pour noter une augmentation significative des grèves depuis 2003. Voir par exemple http://www.gongchao.org/de/texte/2008/pun-ngaiinterview et la carte interactive des grèves compilée par le China Labour Bulletin (CLB) (http://www. numble.com/PHP/mysql/clbmape.html). ONG basée à Hong Kong, CLB est aujourd'hui l'un des premiers acteurs indépendants de la recherche sur le monde du travail et conseille, à travers ses programmes spécifiques d'assistance, un nombre important de travailleurs chinois. 
L'augmentation et la radicalisation des conflits s'expliquent tout d'abord par le hiatus entre le progrès de la législation du travail et l'incapacité des mécanismes institutionnels à réguler les relations professionnelles. En cas de conflit, la loi contraint les travailleurs à passer d'abord par un processus de médiation, puis par un comité d'arbitrage et enfin, en cas d'échec, par les tribunaux. L'explosion, ces dernières années, des recours à ces canaux institutionnalisés de résolution des conflits est cependant trompeuse car ces dispositifs ne répondent que très imparfaitement aux attentes des travailleurs lésés : d'une part, ils sont prévus pour recevoir des plaintes individuelles et non collectives, d'autre part, ils sont notoirement inefficaces.

Ensuite, les attentes et des demandes des travailleurs migrants ont évolué. Les travailleurs de la nouvelle génération, nés dans les années 1980 et 1990, qui représentent déjà plus des deux tiers de la population migrante, sont mieux éduqués et plus qualifiés et n'ont pour la plupart jamais cultivé la terre ${ }^{10}$. Certains ont grandi en ville, d'autres ont émigré avec le désir de profiter de la « vie moderne », de s'épanouir professionnellement et de fonder une famille en zone urbaine. Ils se distinguent en cela de leurs aînés qui se considéraient avant tout comme des paysans occupant un emploi temporaire en ville. La nouvelle génération a subjectivement perdu tout lien avec la campagne et ressent « un sentiment plus profond de colère et d'insatisfaction que la première génération $\gg^{11}$.

Il existe donc à l'heure actuelle une contradiction fondamentale entre les intérêts de travailleurs qui, se plaçant dans la perspective d'une intégration en ville, réclament des salaires au-delà des minima légaux ainsi que l'application des droits sociaux prévus par la loi et les intérêts d'employeurs qui luttent pour maintenir leur compétitivité dans un contexte d'augmentation du coût de la main-d'œuvre depuis $2010^{12}$ et de reprise difficile de l'activité économique depuis la crise financière de 2008. Sur les 30 conflits constituant notre échantillon, 18 concernent des mises à pied, des situations de sous-emplois et de congés forcés non payés (par exemple lorsque l'entreprise ne reçoit plus suffisamment de commandes), des fermetures et des délocalisations d'usines ; 12 concernent le non-respect de la législation par l'employeur (absence de cotisation à la sécurité sociale ou au fonds d'aide au logement, usage illégal du système d'intérim (laowu paiqian), non-paiement ou le sous-paiement des heures supplémentaires, baisses ou retenues de salaires).

\footnotetext{
10. Quanzong Guanyu Xinshengdai Nongmingong Wenti de Yanjiu Baogao (Rapport de recherche de la Fédération nationale des syndicats chinois sur les problèmes relatifs à la nouvelle génération de travailleurs migrants), 2010 (http://www.youngmigrants.org/ew/uploadfile/20100718143155316.pdf).

11. Pun Ngai, Lu Huilin, « Unfinished Proletarianization: Self, Anger, and Class Action among the Second Generation of Peasant-Workers in Present-Day China », Modern China, 36 (5), 2010, p. 512.

12. La grande vague de grèves de l'été 2010 qui a suivi le conflit à Honda Nanhai s'est soldée par une augmentation de $20 \%$ des salaires minimaux dans la plupart des grandes villes de Chine, mais cette augmentation n'a que très imparfaitement compensé la baisse relative des salaires comparée à l'augmentation des bénéfices des entreprises et à l'inflation. Chloé Froissart, « La radicalisation des actions collectives chez les travailleurs migrants et ses conséquences politiques », Chronique internationale de l'IRES, 128, janvier 2011, p. 18-19.
} 


\section{L'ouverture d'un nouvel espace pour la négociation}

Prises de court par l'augmentation des conflits, les autorités centrales ont peu à peu assoupli leur position face aux grèves à partir du milieu des années 2000. Reconnaissant que ces actions exprimaient des demandes économiques légitimes et non une menace politique, elles ont appelé les gouvernements locaux à gérer les conflits « de manière raisonnable » (lixing duidai) en privilégiant le dialogue et non plus la répression ${ }^{13}$. La police n'est désormais autorisée à arrêter des manifestants qu'en cas de « perturbations de l'ordre public », comme un blocage de routes ou la destruction de biens publics ${ }^{14}$. Même si le Parti reste fondamentalement hostile à toute forme d'action collective organisée, cette tendance à la banalisation et à la légitimation de la grève trouve un écho chez certains dirigeants locaux, notamment dans la province du Guangdong, où la municipalité de Shenzhen a publié en 2008 des règlements autorisant les « arrêts de travail » si aucune solution n'avait été trouvée à un conflit majeur au bout de 30 jours ${ }^{15}$.

Tandis que les employeurs sont désormais encouragés à régler les problèmes en interne afin de favoriser la « construction d'une société harmonieuse $»^{16}$, les ouvriers ont acquis un nouveau pouvoir de négociation s'enracinant notamment dans le manque de main-d'œuvre qui affecte les entreprises de manière chronique depuis 2004 dans le delta de la rivière des Perles et du Yangzi ${ }^{17}$. Ils en ont fait une démonstration éclatante lors du conflit à Honda Nanhai à l'été 2010 - au cours duquel les ouvriers ont obtenu une hausse de salaire de $34 \%$ - et de ceux qui ont suivi.

Pour parer à l'augmentation du nombre des conflits, l'idée d'une participation accrue des ouvriers n'a cessé de progresser ces dernières années. Apparaissant comme une nouvelle tactique de gestion de l'instabilité sociale, elle est également portée par l'évolution de la politique économique de la Chine qui, depuis que la crise financière mondiale a entraîné une baisse des exportations, cherche à développer son marché intérieur en augmentant les salaires ${ }^{18}$. Depuis 2010, certaines provinces, notamment le Guangdong, ont ainsi promu au sein des

13. C. Froissart, « The Rise of Migrant Workers' Collective Actions: Toward a New Social Contract in China », dans Khun Eng Kuah-Pearce, Gilles Guiheux (eds), Social Movements in China and Hong Kong. The Expansion of Protest Space, Amsterdam, Amsterdam University Press, 2009, p. 173-174 ; F. Lee Cooke, « New Dynamics of Industrial Conflicts in China: Causes, Expressions and Resolution Alternatives », cité, p. 121.

14. Ching Kwan Lee, Yonghong Zhang, « The Power of Instability: Unraveling the Microfoundations of Bargained Authoritarianism in China », American fournal of Sociology, 118 (6), mai 2013, p. 1491.

15. Shenzhen jingji tequ hexie laodong guanxi cujin tiaoli (Règlements de la zone économique spéciale de Shenzhen pour promouvoir des relations de travail harmonieuses), octobre 2008.

16. F. Lee Cooke, « New Dynamics of Industrial Conflicts in China: Causes, Expressions and Resolution Alternatives », cité, p. 121.

17. En 2004 et en 2010, il manquait 2 millions de travailleurs dans la province du Guangdong. Yinni Peng, « Exit: Power Struggle in a Time of Labor Shortage in South China », Social Transformations in Chinese Societies, $6(1), 2010$, p. 99.

18. Le douzième plan quinquennal (2011-2015) prévoit ainsi dix plans d'action pour améliorer le niveau de vie de ses citoyens, notamment une augmentation moyenne du salaire minimum d'au moins $13 \%$ par an. F. Lee Cooke, « New Dynamics of Industrial Conflicts in China: Causes, Expressions and Resolution Alternatives », cité, p. 125. 
usines la tenue d'élections syndicales qui ont permis aux ouvriers d'élire leurs propres représentants. Cependant, les présidents des syndicats restent nommés par la direction, et ces élections visent avant tout à renforcer la voix des ouvriers dans les syndicats officiels et non à en promouvoir l'autonomisation ${ }^{19}$. Certes, des « négociations collectives » supervisées et menées sous la férule de l'État et du Parti ont été expérimentées à Honda Nanhai et dans la branche de la restauration à Wuhan ${ }^{20}$, mais en dehors du fait qu'elles ne sont pas autonomes, ces expériences sont encore très isolées.

Parallèlement, la notion de négociations collectives (tiji tanpan), introduite à la fin des années 2000 par des avocats militants et des ONG de défense des droits des travailleurs basées à Hong Kong et dans le delta de la rivière des Perles, n'a cessé de gagner du terrain et de faire l'objet de débats, notamment sur le site internet China Collective Bargaining Forum (Fiti tanpan luntan) et la revue Collective Bargaining Research (Fiti tanpan zhidu yanjiu) fondés respectivement en 2010 et 2011 par le cabinet d'avocats Laowei à Shenzhen. Depuis 2010, un ou deux forums nationaux sont organisés chaque année qui réunissent des avocats et des chercheurs spécialistes des questions du travail, des responsables syndicaux, des fonctionnaires et des représentants d'organisations sociales. C'est auprès du réseau d'ONG et d'avocats qui constitue le cœur de ce forum que nous avons mené nos enquêtes.

\section{Les ONG : un nouvel acteur des négociations}

Apparues à la fin des années 1990 pour venir en aide à des travailleurs à l'origine complètement exclus des services urbains et des syndicats, ces organisations ont connu un essor extraordinaire ces dernières années. Selon nos estimations ${ }^{21}$, il y en aurait une quarantaine dans le delta de la rivière des Perles, 10 à Pékin et 5 dans le delta du Yangzi. Ces organisations présentent différents profils et orientations et seules 7 appartiennent au groupe promouvant les négociations collectives que nous étudions. Essentiellement situées dans le delta de la rivière des Perles, ces dernières ont été fondées par des travailleurs migrants qui, ayant rencontré un conflit avec leur employeur, se sont défendus par eux-mêmes. L'une d'entre elles est cependant dirigée à l'heure actuelle par un ancien avocat. Le personnel de ces organisations est composé de travailleurs sociaux et d'anciens leaders ouvriers. Leurs fonds proviennent de fondations et d'ONG étrangères ou hongkongaises, de syndicats ou d'ambassades. Bien que la législation chinoise dénie le droit à la société de s'organiser de manière autonome, ces organisations, enregistrées comme

19. C. Froissart, « L'évolution de la dynamique des grèves en Chine et leur impact sur la démocratisation au sein des entreprises », dans Richard Sobel, Clément Séhier (dir.), Travail, luttes sociales et régulation du capitalisme dans la Chine contemporaine, Villeneuve d'Ascq, Presses Universitaires du Septentrion, à paraître février 2015.

20. Ibid. ; C. King-Chi Chan, E. Sio-leng Hui, «The Development of Collective Bargaining in China: From

"Collective Bargaining by Riot" to "Party State-led Wage Bargaining" », art. cité.

21. Fondées sur les registres tenus par les organisations auprès desquelles nous avons mené nos enquêtes. 
entreprises ou pas enregistrées du tout, parviennent à subsister dans une zone grise non institutionnalisée en raison de l'incapacité des institutions à répondre aux besoins de la population. Considérées comme dangereuses par l'État, qui craint qu'elles ne provoquent une révolution de couleur, elles lui sont pourtant devenues indispensables. En dépit de leur statut incertain et de pressions constantes de la part des autorités, certaines ont réussi au fil du temps à gagner en influence et à élargir leur espace et leur liberté d'action en s'appuyant notamment sur des alliances contingentes avec des membres du Parti ou de l'administration qui trouvent un intérêt dans le fait d'entretenir avec elles des formes de collaboration ${ }^{22}$.

Témoins du désespoir et de la démobilisation suscités chez les travailleurs par les recours institutionnels, ces organisations, à l'origine essentiellement spécialisées dans l'aide juridique et la défense des droits individuels ${ }^{23}$, en sont peu à peu venues à promouvoir la défense des droits collectifs. Si elles ne prétendent pas représenter les ouvriers (soulignant à cet égard qu'elles n'ont pas été élues), elles les aident à élire leurs propres représentants et à négocier par eux-mêmes. Cette position stratégique leur permet par ailleurs de ne pas faire ouvertement concurrence aux syndicats officiels, qu'elles sollicitent régulièrement en faveur des ouvriers. La première tâche des ONG est d'informer très précisément les travailleurs du contenu de la législation et de canaliser leurs émotions. Elles tentent de faire émerger en eux un « moi tactique » face à leur «moi émotionnel $»^{24}$, en leur apprenant à formuler leurs demandes de manière rationnelle et pondérée, par d'autres moyens que les arrêts de travail et les pétitions, pour ne pas entraîner la répression. La seconde tâche consiste à « transformer un rassemblement temporaire en une solidarité de groupe stable $»^{25}$. Les ONG apprennent aux travailleurs à élire leurs représentants, sur candidature spontanée ou sur leur propre proposition. Dans tous les cas, il y a au moins un représentant par atelier que compte l'usine. Les élections sont organisées soit dans les dortoirs de l'usine, soit dans les locaux de l'ONG. Les représentants se voient ensuite attribuer différents rôles : certains sont chargés de négocier directement avec l'employeur, d'autres d'informer et de mobiliser les employés, notamment grâce à la création d'un groupe de messagerie instantanée $\mathrm{QQ}^{26}$ qui se révèle très utile pour coordonner l'action au sein de

22. Anthony J. Spires, « Contingent Symbiosis and Civil Society in an Authoritarian State: Understanding the Survival of China's Grassroots NGOs », American fournal of Sociology, 117 (1), 2011, p. 1-45 ; Joseph Y. S. Cheng, Kinglun Ngok, Wenjia Zhuang, « The Survival and Development Space for China's Labor NGOs: Informal Politics and Its Uncertainty », Asian Survey, 50 (6), 2010, p. 1082-1106.

23. Xu Yi, « Labor Non-governmental Organizations in China: Mobilizing Rural Migrant Workers », fournal of Industrial Relations, 55 (2), 2013, p. 243-259.

24. Sur les notions de «moi émotionnel » et de «moi tactique », voir James C. Scott, La domination et les arts de la résistance : fragments du discours subalterne, Paris, Éditions Amsterdam, 2008 ; et notamment l'excellente analyse qu'en fait Christian Lazzeri, Pouvoir, rationalité et résistance : autour de la théorie politique de fames C. Scott (http://sophiapol.hypotheses.org/14654).

25. Entretien, Canton, avril 2014.

26. Détenu par le géant des nouvelles technologies chinois Tecent, QQ est le programme de messagerie instantanée gratuit le plus populaire et compte aujourd'hui plus de 100 millions d'utilisateurs. 
l'usine et maintenir la cohésion parmi les ouvriers dont le nombre peut parfois atteindre plusieurs milliers. Certains représentants sont en charge des relations avec l'ONG ou le cabinet d'avocats qui suit l'affaire, d'autres de la communication avec l'extérieur, notamment avec les médias.

L'ONG aide ensuite les travailleurs à rédiger et signer une lettre de revendications, c'est-à-dire à établir une hiérarchie entre les demandes, quitte à en écarter certaines. Ce document sert à informer la direction de la demande de négociation et de la teneur des revendications, et constitue la base d'une lettre ouverte postée sur les réseaux sociaux pour informer l'opinion publique et recueillir des soutiens. L'unité et la solidarité sont également encouragées par la création d'un fonds géré par un ou deux représentants, et qui sert à couvrir les frais de transport, de repas, de téléphone, d'impression, de photocopie, d'envoi postal, et à compenser la perte de revenus liée à l'action collective. Dans la mesure où chacun est tenu de payer pour celle-ci, tout le monde se sent impliqué. Selon les termes employés par les ONG, cette stratégie vise à empêcher le «free riding » (da bianche).

L'ONG entretient une discussion permanente avec les représentants des travailleurs et les aide à établir une stratégie d'action - qui commence par la réunion et la préparation de documents - au cours de séances qui se tiennent régulièrement dans ses locaux. Elle leur apprend que la minorité doit toujours suivre la majorité et qu'il ne faut pas disséminer des informations tant qu'ils ne se sont pas mis d'accord. Elle leur fait comprendre la nécessité de renoncer à leurs intérêts personnels pour que chacun obtienne quelque chose collectivement. Elle les met en contact avec des journalistes mais aussi avec des travailleurs qui ont vécu un conflit similaire au leur afin qu'ils puissent être encouragés par leurs succès mais aussi apprendre de leurs erreurs.

Avec l'aide des ONG et des avocats, les travailleurs parviennent à rééquilibrer les rapports de force entre capital, travail, syndicats et autorités locales et tentent de promouvoir à plus long terme l'émergence d'un système de négociations collectives.

\section{Types de ressources et de stratégies : les leviers de la négociation}

\section{Une résistance rationnelle}

Le premier défi auquel sont confrontés les travailleurs est d'amener l'employeur à la table des négociations. Celui-ci ignore généralement la lettre des ouvriers ou répond d'emblée qu'il ne peut satisfaire leurs demandes. S'il accepte de recevoir les représentants, il refuse souvent la négociation en réservant sa réponse pendant de longues semaines, voire de longs mois. Tout l'enjeu pour les représentants des travailleurs est alors de trouver un équilibre entre la pression, qui permet de rééquilibrer les rapports de force, et l'autolimitation, qui permet de se protéger de la répression. 
Dans son étude sur la mobilisation des propriétaires de logement contre les politiques d'incinération des déchets à Canton, Amy Zhang souligne que la mise en avant par les protestataires d'une « résistance rationnelle »- se distinguant à la fois d'une résistance émotionnelle et violente, mais aussi d'une résistance politique - est devenue un nouveau procédé rhétorique permettant de s'opposer dans un système autoritaire et de créer de nouveaux modes de résolution des conflits fondés sur le dialogue et la négociation ${ }^{27}$. Les représentants des travailleurs font valoir que leurs demandes et l'action en elle-même sont « justes, raisonnables, légales » (zhengyi, heli, hefa). Ils s'appuient pour ce faire sur les textes de loi ainsi que sur les preuves qu'ils ont pu réunir (contrat, feuille de paie, etc.). Ils ont par ailleurs pour mot d'ordre de garder leur sang-froid quoi qu'il arrive, de parler de manière pondérée et à tour de rôle. Chacun est chargé d'aborder une question spécifique, qui transmettant les demandes des ouvriers, qui évoquant l'état d'esprit de ces derniers, qui insistant pour que l'employeur s'engage à respecter le protocole des négociations, lui signifiant par exemple qu'aucun représentant n'est fondé à négocier seul à seul avec lui et que toute négociation doit nécessairement impliquer l'ensemble des représentants, ou lui demandant de s'engager par écrit à ne pas « faire les comptes après la moisson » (faire subir des représailles aux représentants). S'ils demandent que la direction leur fournisse des « explications rationnelles $\gg$ pour des décisions qui leur paraissent arbitraires, les travailleurs veillent également à ne pas, dans un premier temps, acculer l'employeur. Ils font valoir qu'ils se mettent à sa place en restreignant leurs demandes (quitte à les augmenter par la suite) et que ses intérêts rejoignent ceux des employés.

Ainsi, les travailleurs de Gaoya, une entreprise fabriquant des bijoux à Canton, ont demandé à leur employeur, un entrepreneur hongkongais, pourquoi il ne payait pas la sécurité sociale, ne leur donnait pas de travail à faire et préférait sous-traiter alors qu'ils étaient payés à la pièce. Lorsque celui-ci leur a répondu qu'il voulait réduire les coûts, les représentants « l'ont aidé à faire ses comptes » et lui ont fait valoir que sa tactique lui faisait perdre de l'argent car il rémunérait des ouvriers extérieurs pour faire le travail tout en payant le salaire de base de ses propres ouvriers et les frais de fonctionnement de l'usine. Ils ont ainsi réussi à le persuader de n'avoir recours à la sous-traitance que pour le travail que ses employés ne pouvaient pas faire. De même, 20 ouvriers se partageaient le travail de 4 personnes car l'employeur ne voulait pas payer des primes de licenciement (équivalentes à un mois de salaire par année travaillée). Or le salaire était tellement bas que les ouvriers souhaitaient démissionner, tout en obtenant une compensation. Les représentants ont alors proposé que 10 personnes partent et ont négocié une compensation s'élevant à trois mois de salaire pour cinq années travaillées ${ }^{28}$.

27. Amy Zhang, «Résistance rationnelle : mobilisation des propriétaires contre l'incinération des déchets à Canton », Perspectives chinoises, 2, 2014, p. 50.

28. Entretien, Canton, avril 2014. 


\section{Faire pression sans encourir la répression}

L'action collective n'est donc pas organisée de manière conflictuelle, les travailleurs étant soucieux de montrer qu'ils privilégient le dialogue et le compromis. Ainsi, ils posent des limites à leur action en refusant la violence (le mot d'ordre est de ne pas casser les machines, de ne pas détériorer les lieux ou le matériel) et en maintenant l'action collective dans l'enceinte de l'usine pour en limiter la visibilité. Tout en se conformant aux règles définies par le Parti, ils ont recours à une palette d'actions qui visent à empêcher le fonctionnement normal de l'entreprise tout en limitant les risques de répression : arrêter le travail (mais poinçonner les cartes de présence), occuper les entrepôts ou l'entrée principale de l'usine, faire le siège du bureau du personnel, encercler le directeur général ou son représentant légal, manifester au sein de l'usine en déployant des banderoles et en scandant des slogans, faire le plus de bruit possible (comme jouer de la flûte) dans les ateliers pour entraver la production et inviter les ouvriers à se joindre à l'action.

Grâce aux réseaux sociaux, l'action des travailleurs parvient néanmoins à franchir les frontières de l'usine et à trouver d'autres formes de visibilité et de publicité. Aujourd'hui, les travailleurs ont un accès beaucoup plus aisé à Internet grâce à leur téléphone portable. La plupart ont un compte de messagerie instantanée QQ qui comprend également une partie « open space » de type Facebook dont ils se servent pour publier des informations sur les conflits. Les représentants utilisent cette plateforme pour informer les médias et attirer l'attention des autorités (qui y ont recours, elles aussi, pour prendre le pouls de l'opinion), notamment en leur transmettant leurs lettres ouvertes et leurs demandes de négociation. Les ONG s'en servent également pour relayer les informations concernant les conflits et transmettre les demandes des travailleurs aux autorités locales qui, parfois, accueillent d'autant mieux ces derniers lorsqu'ils viennent déposer leur plainte, notamment lorsque le conflit a déjà fait l'objet d'articles dans la presse. $\mathrm{Si}$ la grève ou la menace de grève permet quelquefois de résoudre rapidement le conflit (notamment lorsque l'employeur a des commandes à honorer), il existe cependant des situations - comme les relocalisations, les licenciements, les fermetures d'usines - où les arrêts de travail ne sont pas efficaces. Les ouvriers de l'usine de meubles Liansheng à Canton ont ainsi fait grève pendant 57 jours sans succès : l'entreprise voulait acculer ses employés à la démission pour réduire ses coûts de production et avait recours à la sous-traitance extérieure. Dans ces cas-là, le soutien du Parti ou de l'État est nécessaire à la résolution du conflit.

Malgré la défiance des ouvriers envers les syndicats, les ONG tentent de persuader les représentants d'aller systématiquement trouver ces derniers pour leur remettre la lettre de revendications et leur demander d'intervenir auprès de l'employeur ou de superviser les négociations. Cette stratégie sert plusieurs objectifs : jouer le syndicat du district contre le syndicat de l'usine qui est désavoué (lorsqu'il existe), officialiser les représentants ouvriers et formaliser une plateforme de dialogue, 
légitimer le rôle des ONG et des représentants ouvriers en montrant qu'ils associent les syndicats à la résolution des conflits au lieu de leur faire concurrence et, enfin, contraindre ces derniers à endosser leurs responsabilités. Elle consiste ainsi à retourner le « texte public $»^{29}$ contre lui-même de manière très habile : les ONG considèrent que si les syndicats ne répondent pas, ce sont eux qui sont dans leur tort et l'État ne peut pas accuser les ouvriers de s'être défendus par eux-mêmes. Porter plainte auprès des syndicats et des autorités locales constitue parfois une gradation de l'action et une occasion de réviser les demandes à la hausse. Confrontés au refus de l'entreprise Gaoya de payer ses arriérés de cotisations à la sécurité sociale, les représentants ouvriers ont sollicité le syndicat de la ville, le bureau des ressources humaines et de la sécurité sociale du district de Panyu à Canton ainsi que l'équipe d'inspection du travail. En plus du versement des sommes dues à la sécurité sociale, ils ont demandé une hausse des salaires ainsi que, conformément à la loi, des congés payés et des visites médicales gratuites régulières.

Les ouvriers de l'entreprise Yanlian, une entreprise étatique d'extraction de gaz et de pétrole située à Yan'an dans la province du Shaanxi, avaient été contraints par leur employeur de signer des contrats illégaux d'intérim qui permettaient à ce dernier de verser des salaires moins élevés et d'échapper au paiement des primes d'ancienneté et de compensations élevées en cas de licenciement. Les employés demandaient la signature de CDI et l'harmonisation des salaires. Confrontés pendant quatre mois au refus de l'employeur de négocier, ils lui ont envoyé, sur les conseils du cabinet d'avocats Laowei, un préavis de grève, spécifiant que celle-ci était motivée par son refus de la négociation. Ils ont transmis leurs revendications et une description détaillée de la situation aux autorités provinciales ainsi qu'aux fédérations syndicales municipale et provinciale en les priant d'intervenir. Enfin, ils ont transmis leur lettre de revendications aux médias via leurs réseaux sociaux. Ils n'ont finalement pas eu à mettre à exécution leur menace de grève : leurs actions ont forcé les syndicats à intervenir, et la pression sur l'employeur a été suffisante pour qu'il accepte les négociations. La direction a aboli le système d'intérim, mais s'est vengée ultérieurement sur les représentants ouvriers en les licenciant ou en les affectant à des postes subalternes.

Si le succès des négociations est encore en grande partie tributaire de l'intervention du Parti et de l'État, il reste très difficile de gagner le soutien des autorités locales, qui sont souvent d'autant plus complices des employeurs qu'elles cherchent à freiner les délocalisations. Sur les trois conflits concernant le paiement de la sécurité sociale dans des entreprises privées que nous avons suivis, l'employeur avait à chaque fois été autorisé par les autorités locales à ne cotiser que pour une partie de ses employés (respectivement $10 \%, 20 \%$ et un tiers). Les employés de l'entreprise de bijoux Gaoya à Canton n'ont pu obtenir le paiement des arriérés de cotisations avant 2004 : le responsable du bureau du travail du district a couvert

29. J. C. Scott, La domination et les arts de la résistance : fragments du discours subalterne, op. cit.. 
l'entreprise, faisant croire qu'il ne pouvait retrouver aucune donnée sur celle-ci avant 2004, alors même que les représentants lui faisaient valoir qu'elle s'était ré-enregistrée sous un autre nom.

Pour parer aux blocages des autorités au niveau local, les protestataires tentent d'exploiter les divisions au sein de l'administration et sollicitent l'aide des échelons supérieurs, mais ils sont souvent accueillis avec hostilité et indifférence, les cadres faisant traîner indûment les procédures ou les renvoyant aux échelons inférieurs sous prétexte qu'il revient à ces derniers de régler le problème. La résolution du conflit dans l'entreprise Hengbao, sur lequel nous reviendrons, a ainsi pris plus d'un an à cause de l'indifférence et de l'impéritie de l'administration.

\section{Un équilibre très instable}

La limite à l'action collective est souvent difficile à trouver lorsque les employeurs ne veulent rien entendre et que les autorités locales et les syndicats refusent leur soutien aux grévistes, d'autant que les employeurs ont la loi de leur côté et peuvent licencier les représentants ouvriers au motif qu'ils ont « sévèrement porté atteinte aux règlements de l'entreprise » ou ont « enfreint la discipline » (Loi sur les contrats de travail, art. 39). Le 9 décembre 2011, après plus de sept mois de négociations, la direction de Hengbao, une PME fabriquant des bijoux à Canton, a accepté officiellement de payer ses arriérés de cotisation à la sécurité sociale, tout en exigeant que les employés paient leur part avant le 31 décembre. Les employés se sont exécutés, bien que pour certains le montant de la cotisation s'élevât à plus de six mois de salaire. Trois mois plus tard, l'entreprise n'ayant toujours pas payé sa part, plus de 130 ouvriers se sont postés devant le bureau du représentant légal de l'usine, l'empêchant de sortir jusqu'à ce qu'il donne une date de paiement. Au bout de deux jours, des heurts ayant éclaté, la police est intervenue et a placé les deux représentants en chef en détention pendant 25 jours pour avoir « incité les ouvriers à séquestrer illégalement » le responsable du département des ressources humaines.

En mai 2013, un groupe de 126 infirmières et agents de sécurité de l'hôpital public de médecine chinoise de Canton ont déposé une plainte auprès de la fédération syndicale municipale pour dénoncer l'absence de contrat de travail et de sécurité sociale, des salaires inégaux à travail égal et le non-paiement des heures supplémentaires. En août, un accord a été trouvé entre la direction et les infirmières, excluant les agents de sécurité. Un groupe de 12 d'entre eux a alors continué le mouvement en montant sur le toit de l'hôpital pour y déployer une banderole ${ }^{30}$. Ils ont été arrêtés, accusés de « perturbation de l'ordre social » et formellement jugés en avril 2014 après huit mois d'emprisonnement.

30. http://www.clb.org.hk/en/content/hospital-security-guards-detained-50-days-after-stagingprotest. 


\section{Refaçonner les relations entre l'État et la société \\ Contrecarrer les stratégies de « maintien de l'ordre social » des autorités locales}

Les autorités locales peuvent être favorables à une résolution négociée des conflits lorsqu'elles y voient un moyen de réduire les grèves et le nombre de plaintes devant les comités d'arbitrage et les tribunaux, partant le degré d'instabilité sociale qui constitue l'un des critères fondamentaux sur lesquels elles sont évaluées par leur hiérarchie. Les syndicats sont également divisés. Si certains de leurs cadres sont favorables à l'avancement du droit de grève et des négociations collectives, d'autres s'y opposent fermement. D'une manière générale, les syndicats sont plus enclins à intervenir en faveur des employés de multinationales que des employés d'entreprises locales ou de PME taïwanaises ou hongkongaises.

L'ouverture d'esprit dont témoignent certains cadres est encore rare. Cela est particulièrement vrai des autorités au niveau hiérarchique le plus bas, celui du comité de rue et du district, qui sont les premières responsables en matière de maintien de la stabilité sociale, et auxquelles les protestataires doivent s'adresser en premier. Leur réflexe, lorsqu'elles sont sollicitées, est de demander aux travailleurs de cesser toute action collective et d'avoir recours aux canaux institutionnalisés de résolution des conflits, en premier lieu les comités de médiation et d'arbitrage. Les ONG ne sont pas dupes du fait que ces recours aux comités ne sont qu'« un jeu juridique et bureaucratique invoqué par les cadres pour absorber les troubles $\gg^{31}$. Aussi veillent-elles à ce que les travailleurs ne tombent pas dans le piège, ne serait-ce que parce qu'il existe des cas où l'arbitrage ne constitue pas un recours possible : par exemple lorsque l'entreprise fait faillite ou est relocalisée et que l'employeur n'est pas joignable, ou lorsque le conflit va au-delà de ce qui est prévu par la loi. De façon générale, elles préviennent les ouvriers que s'engager dans de telles procédures signifie perdre une énergie et un temps précieux dans des paperasseries sans fin, des attentes interminables et des appels réitérés pour un résultat très aléatoire.

Les ONG tentent également de prendre à revers les stratégies de maintien de la stabilité sociale déployées par les autorités locales, notamment en répondant partiellement à leurs attentes pour pouvoir mieux les dévoyer. Selon Lee et Zhang, ces stratégies sont au nombre de trois ${ }^{32}$. La première consiste à évaluer la gravité de la contestation en catégorisant l'incident en fonction du nombre de protestataires et du degré d'émotion manifesté, du coût financier impliqué et du potentiel de contagion. Le premier objectif des autorités - tout comme des ONG - est

\footnotetext{
31. Ibid..

32. Ching Kwan Lee, Yonghong Zhang, « The Power of Instability: Unraveling the Microfoundations of Bargained Authoritarianism in China », art. cité, p. 1486.
} 
de contrôler l'émotion des protestataires et de la canaliser vers une discussion rationnelle pour éviter que le conflit ne s'envenime. Or, tandis que les autorités pensent qu'« empêcher les protestataires de provoquer des scènes d'instabilité et de désordre constitue un puissant levier [en leur faveur à elles] dans le processus de négociation $\gg^{33}$, les ONG font le pari que la manière posée et formelle avec laquelle les travailleurs sollicitent les autorités obligera à terme ces dernières à répondre de même et à intervenir dans les conflits de manière réellement neutre et constructive. Les ONG pensent que la confiance qu'elles tentent de susciter chez les ouvriers envers les syndicats et les autorités locales finira par faire naître chez ces derniers un nouveau sens de leurs responsabilités. Cette stratégie porte parfois ses fruits. Dans le cas de l'usine Liansheng, les autorités locales, après avoir épuisé toutes les tactiques habituelles pour venir à bout du conflit, ont supervisé plus de vingt rounds de négociations entre l'employeur et les employés entre juillet et octobre 2013. Elles ont ainsi permis la signature d'un contrat collectif interdisant toute baisse des primes et subventions allouées par l'entreprise visant à compenser les hausses du salaire minimum. Face au refus de l'employeur d'attribuer à tous les employés une rémunération égale à travail égal, les autorités locales ont fini par créer un comité $a d$ hoc de règlement du conflit qui a débouché pour les travailleurs sur un plan social sans précédent.

La deuxième stratégie des autorités est de « trouver le point d'accès à la foule », c'est-à-dire d'identifier des leaders. Si personne ne se présente spontanément, elles demandent que des représentants soient élus. Les ONG présentent, elles aussi, les élections de représentants qu'elles supervisent comme le seul moyen de conduire un dialogue social, de prévenir un incident de masse et de résoudre rapidement le conflit, autrement dit de maintenir la stabilité sociale. Autorités locales et ONG s'accordent donc sur la nécessité d'agréger les intérêts pour que les demandes soient exprimées de manière ordonnée et de créer des intermédiaires entre l'État et les protestataires. Mais tandis que, pour les autorités locales, l'élection de représentants constitue le début d'un processus de division et de cooptation ${ }^{34}$, tout l'enjeu pour les ONG est de parvenir à maintenir l'unité et la solidarité parmi les travailleurs. Les représentants sont en effet constamment l'objet de tentatives de corruption ou de menaces de la part des autorités locales comme des employeurs. Ces derniers tentent également de diviser les travailleurs en proposant des solutions qui en avantagent certains - notamment les représentants - et en lèsent d'autres.

Dans le cas de l'usine Hengbao, la direction a fini par accepter de rembourser ses arriérés de cotisations à la sécurité sociale, mais seulement à partir de 2004, date à laquelle le négociateur en chef avait été employé par l'entreprise. Dans la mesure où de nombreux employés travaillaient dans l'usine depuis plus de dix ans, ce dernier a refusé la proposition de la direction et a continué le combat. 
Selon son propre témoignage, il a gagné le respect des ouvriers et d'autres se sont joints à l'action collective. En revanche, dans le cas du conflit à Hitachi Metal, une usine japonaise de composants magnétiques à Canton, qui portait également sur le paiement des arriérés de cotisations à la sécurité sociale, la direction a obtenu à plusieurs reprises la défection des représentants, obligeant chaque fois les ouvriers à en élire de nouveaux. Finalement, elle n'a accepté de payer la sécurité sociale que pour ceux qui admettaient qu'ils étaient ou avaient été représentants, décapitant ainsi le mouvement ${ }^{35}$.

Enfin, la troisième stratégie des autorités locales consiste à « transformer les droits des citoyens tels qu'ils sont stipulés par les textes de loi en droits réalistes et réalisables en fonction des circonstances $\gg^{36}$. Aussi n'existe-t-il pas pour les autorités de droits intangibles mais seulement une « construction conjointe des droits » dans la pratique, en fonction de considérations pragmatiques. Si les ONG encouragent dans un premier temps les ouvriers à hiérarchiser leurs demandes et à les circonscrire à « ce qui est raisonnable et légal », quitte à favoriser parfois les compromis, leur objectif est avant tout d'aider les travailleurs à obtenir l'application du droit du travail, voire ce qui n'est pas prévu par la loi. Elles les incitent à mener les négociations jusqu'au bout et à ne pas transiger sur leurs droits, par exemple à ne pas accepter une prime de licenciement bien inférieure à ce qui est prévu par la législation. L'une d'entre elles a ainsi poussé les employées d'un des comités de rue du district de Panyu à Canton chargées de l'hygiène et de la propreté à demander une hausse de salaire alors qu'elles avaient déjà obtenu de leur employeur le paiement des arriérés de cotisations à la sécurité sociale. Elles préféraient cumuler les emplois plutôt que d'« offenser » leur employeur en formulant une autre demande. L'ONG leur a expliqué que cette revendication était légitime puisque le droit à un salaire minimum est inscrit dans la loi. Les ONG insistent également dans leurs formations sur le fait que la législation ne prévoit que le minimum et que les travailleurs sont fondés à demander plus car celui-ci ne correspond pas nécessairement à ce qui est juste et raisonnable. Elles leur transmettent ainsi l'idée que les négociations visent non seulement à protéger leurs droits mais aussi leurs intérêts.

\section{Refonder les syndicats au sein de l'usine}

Si elle consiste dans un premier temps à changer la nature des syndicats aux niveaux supérieurs à l'entreprise en les impliquant dans la résolution des conflits et en essayant de susciter la confiance de part et d'autre, la stratégie des ONG vise dans un second temps à s'appuyer sur ces échelons supérieurs pour promouvoir

35. Entretiens avec les représentants ouvriers, Canton, avril 2014.

36. Ching Kwan Lee, Yonghong Zhang, « The Power of Instability: Unraveling the Microfoundations of Bargained Authoritarianism in China », art. cité, p. 1490. 
des élections syndicales au niveau de l'entreprise. Celles-ci sont en effet perçues par les ONG comme par les travailleurs que nous avons rencontrés comme le seul moyen non seulement de parer à l'arbitraire patronal mais aussi de protéger les représentants en leur permettant d'acquérir un statut légal pour négocier avec l'employeur. Cependant, si l'organisation d'élections directes a bien eu lieu sous la férule du Parti après le conflit de Honda Nanhai en 2010, les initiatives de la base rencontrent beaucoup moins de succès. Les représentants de Hengbao et de Hitachi n'ont cessé, en vain, de transmettre des pétitions aux échelons syndicaux supérieurs et aux autorités locales pour demander la tenue d'élections. L'expérience de Zhu Xiaomei, représentante en chef des ouvriers d'Hitachi, illustre les espoirs et les déboires que suscite toute volonté de refonder les syndicats de base. Après quatre mois de négociations, la direction a accepté en août 2013 de payer ses arriérés de cotisations à la sécurité sociale pour une partie de ses salariés, mais a transféré Zhu d'un poste d'organisation de la production à un poste subalterne d'archiviste, avec une caméra de surveillance au-dessus de son bureau ${ }^{37}$. Tout en portant plainte auprès d'un comité d'arbitrage pour rupture de contrat, elle est allée trouver le syndicat du district en octobre 2013 pour demander l'organisation d'élections syndicales, l'entreprise étant dépourvue de syndicat. Sous la pression du syndicat de la municipalité, le syndicat du district a finalement accepté d'inciter l'employeur à organiser des élections mais celui-ci a licencié Zhu de peur qu'elle ne soit élue présidente du syndicat, et a finalement instauré un syndicat acquis à l'entreprise ${ }^{38}$. Il va de soi que ce type d'expérience ne peut que briser la confiance des ouvriers envers les syndicats et semble contrecarrer pour le moment la stratégie des ONG.

\section{Les résultats des négociations}

Le résultat dépend de la persévérance et du degré de cohésion que les ouvriers arrivent à maintenir. Les employeurs parviennent parfois à diviser les grévistes au point que ceux-ci n'obtiennent même pas le minimum légal, notamment dans les cas de fermeture ou de délocalisation d'usines où les ouvriers acceptent des compensations très médiocres parce qu'ils savent que la relation avec l'employeur va de toute façon être rompue. Sur les 30 conflits qui constituent notre échantillon, 19 (soit $63 \%$ ) ont échoué parce que les employeurs ou les autorités ont réussi à diviser les grévistes, mais cela signifie également que dans 37 \% des cas les négociations ont permis aux travailleurs d'obtenir des conditions conformes à la législation, voire meilleures que ce que prévoit la loi. Ainsi, certaines entreprises comme Gaoya se sont complètement remises aux normes du droit du travail chinois : l'entreprise a payé ses arriérés de cotisations à la sécurité sociale depuis 2004, les employés ont

37. http://www.clb.org.hk/en/content/hitachi-sacks-worker-activist-who-lobbied-trade-union.

38. Entretien, Canton, avril 2014. 
signé un CDI avec l'employeur, ont obtenu des congés payés, un examen médical gratuit par an, la garantie d'une compensation en cas de rupture de contrat, et les ouvrières ont obtenu un congé maternité. Parfois, les négociations profitent à toute une branche d'activités. Lorsque les employées chargées de l'hygiène et de la propreté d'un des comités de rue du district de Panyu à Canton ont obtenu le paiement de leurs arriérés de cotisations à la sécurité sociale, une augmentation de salaire, un jour de repos par semaine, des congés annuels payés et un triplement de leur salaire en cas de travail pendant les congés prévus par la loi, des employées d'autres quartiers se sont mises en grève pour revendiquer les mêmes conditions de travail et ont obtenu de la municipalité de Canton une augmentation substantielle de leurs salaires et de leur couverture sociale en janvier 2013. Enfin, le résultat des négociations va parfois bien au-delà de ce qui est prévu par la loi, comme dans le cas de l'usine Liansheng. La direction sous-traitait à l'extérieur pour forcer les ouvriers - payés à la pièce - à poser leur démission afin de réduire les coûts salariaux de restructuration. Déterminés à démissionner, les employés voulaient néanmoins être indemnisés. Après 70 jours de lutte acharnée, ils ont conclu en octobre 2013 un plan social sans précédent leur donnant une indemnité à hauteur de $70 \%$ du salaire mensuel par année travaillée calculée sur la base du mois de l'année précédente où le salaire avait été le plus élevé, soit presque aussi bien qu'une indemnité de licenciement qui s'élève selon la législation à un mois de salaire par année travaillée. En général, le résultat des négociations est sanctionné par un accord en bonne et due forme signé entre les représentants et la direction, et approuvé par l'ensemble des employés.

\section{Une difficile institutionnalisation}

Les ONG et le cabinet d'avocats Laowei sont également des « entrepreneurs de politique publique $\gg$ (policy entrepreneurs ${ }^{39}$ ) qui tentent d'influencer la législation par un travail de plaidoyer auprès des autorités au niveau local. Pour ce faire, ils présentent les négociations collectives comme un processus non politique permettant de maintenir la stabilité sociale ${ }^{40}$. Leur but est toutefois de promouvoir le droit de grève et d'obtenir des garanties juridiques pour la protection des représentants des travailleurs. Certaines de leurs propositions ont été retenues, notamment dans les avant-projets de Règlements de la province du Guangdong pour la gestion démocratique des entreprises et de Règlements sur les consultations collectives dans la zone économique spéciale de Shenzhen rendus publics pour discussion en août 2010. Ces deux textes constituaient un pas important vers l'institutionnalisation du droit de grève et de véritables négociations collectives,

39. John W. Kingdon, Agendas, Alternatives, and Public Policies, New York, Harper Collins, 1995 (2éd.), p. 122-123. 40. Voir par exemple http://www.clb.org.hk/en/content/wall-street-journal-china-journal-labor-activistwhy-china-needs-collective-bargaining-now. 
puisqu'ils établissaient par exemple des procédures de formation de comités de représentation des employés dont les pouvoirs étaient clairement définis. Un employeur pouvait être contraint à engager une négociation collective sur les salaires si au moins un tiers des employés le demandaient et une consultation sur les salaires devait être organisée au moins une fois par an. Si l'employeur ne répondait pas à cette demande dans les quinze jours et qu'une grève en résultait, il n'était pas autorisé à licencier les grévistes. Enfin, les employeurs ne donnant pas suite à ce qui avait été décidé lors des négociations pouvaient être soumis à une amende de 50000 yuans. Ces deux textes ont été mis en échec à l'automne 2010, tant en raison de la réticence de certains cadres que de l'opposition farouche des chambres de commerce hongkongaises ${ }^{41}$. Les mêmes raisons expliquent l'échec de l'avant-projet de Règlements de la province du Guangdong sur les consultations collectives et les contrats collectifs rendus publics en octobre 2013. Ces textes reconnaissaient le droit des travailleurs à élire leurs propres représentants pour « discuter » (xieshang) avec la direction lorsque l'entreprise n'avait pas de syndicat ou que celui-ci était dans l'incapacité de remplir ses responsabilités, ainsi que leur droit à mandater un agent extérieur pour les représenter dans les discussions avec l'employeur. Enfin, ils reprenaient le point des règlements de 2010 selon lequel tout arrêt de travail faisant suite au refus de l'employeur de négocier ne pouvait donner lieu à une rupture du contrat de travail. Même s'ils ne sont pas passés, ces textes témoignent du degré d'avancement de la réflexion et du fait qu'une partie des autorités est déjà acquise aux négociations collectives.

Pour combler ce vide législatif, inspirer la législation à venir et fournir un guide pratique aux employeurs et aux salariés, les activistes ont publié en octobre 2013 un code des négociations collectives ${ }^{42}$ qui se présente comme la synthèse et la formalisation de toutes les expériences de négociations menées jusqu'à maintenant. Largement diffusé sur Internet et les réseaux sociaux, ce document a été également transmis aux autorités compétentes au niveau national et provincial. Ses 65 articles détaillent les méthodes pour initier, préparer, conduire, conclure des négociations et superviser la mise en œuvre des accords. Il s'agit d'un texte très pondéré, qui mentionne autant les droits des employés que ceux des employeurs, et vise à favoriser la confiance entre toutes les parties. Il énonce les conditions dans lesquelles les travailleurs sont fondés à se mettre en grève mais souligne qu'il ne peut y avoir recours à la grève que lorsque tous les autres moyens ont échoué. Il reconnaît également le droit des travailleurs d'avoir recours à un tiers (ONG, avocat) si les négociations sont dans l'impasse. Il implique par ailleurs la marginalisation des syndicats puisqu'il stipule que le comité de négociation est

41. « Guangdong ni lifa yi gongchao, gongren yi qiguan, gangshang lianshu fandui » (Le Guangdong étudie la possibilité de légiférer pour restreindre la vague de grèves, les entrepreneurs hongkongais unis dans l'opposition) (http://www.boxun.com/news/gb/china/2010/09/201009170537.shtml).

42. Une traduction en anglais de ce texte est disponible sur http://www.clb.org.hk/en/sites/default/files/ File/A\%20Code\%20of\%20Collective\%20Bargaining.pdf. 
formé par les représentants des travailleurs et des membres du comité syndical de l'usine, mais que le poids de ces derniers ne peut dépasser un tiers des sièges. En cas d'absence de syndicat sur le lieu de travail, le code fournit aux employés une procédure détaillée pour informer de leur action le syndicat de leur district et lui donner la possibilité de s'investir dans le processus de négociation.

Aujourd'hui, l'État et le Parti chinois ne sont plus tout puissants : la réintroduction de l'économie de marché et du droit a fait naître des demandes et des contradictions sociales que les institutions ne peuvent ni satisfaire ni absorber. Le Parti a beaucoup à perdre, tant sur le plan économique que politique, s'il ne parvient pas à juguler les révoltes ouvrières et à s'assurer de la coopération de la population. S'engouffrant dans cette brèche, les ONG et le cabinet d'avocats que nous avons étudiés proposent une autre vision de l'harmonie sociale, fondée non plus sur la capacité du Parti à synthétiser et réconcilier l'ensemble des intérêts divergents mais sur celle de chaque partie à s'organiser de manière autonome pour parvenir à des solutions de compromis. C'est ce dont témoigne aujourd'hui l'émergence de véritables négociations collectives.

Comme dans tout système coercitif, il existe en Chine un texte public et des textes cachés qui lui répondent et témoignent d'une utilisation stratégique des normes afin d'éviter la confrontation ouverte nécessairement vouée à l'échec. S'ils sont certes contraints de s'autolimiter dans leurs actions pour se conformer à ce texte public, les ouvriers et les ONG participent également à l'élaboration d'un nouveau code - devenu explicite en octobre 2013 - qui fait l'objet d'une négociation permanente et tend à redéfinir les rapports entre les employeurs, les employés et les syndicats. La négociation ne porte pas seulement sur ce qui est négocié mais aussi sur la manière de négocier. Plus fondamentalement, l'idée même de négociation est désormais acquise dans un système qui, en dépit de ses fondements politiques et idéologiques, est aujourd'hui amené à reconnaître, du moins dans la pratique, l'existence d'intérêts antagonistes, partant, l'évolution progressive des modes de représentation et de gestion des conflits. Bien que - ou du fait que - son ressort stratégique essentiel soit d'éviter tout défi ouvert au monopole du Parti communiste sur la sphère politique et de faire valoir l'existence d'intérêts partagés entre ce dernier et la société, la mobilisation des acteurs sociaux représente un pouvoir d'innovation et un impact politique indéniables.

Cependant, comme en témoignent les difficultés d'institutionnalisation des négociations collectives, son impact concerne plus, à l'heure actuelle, les rapports entre l'État et la société que le système politique en lui-même. Le Parti se garde de systématiser ces expériences de négociations afin qu'elles restent des formes de résolution ad hoc et localisées des conflits, comme autant de respirations lui permettant d'assouplir le fonctionnement du système pour mieux en préserver la nature. Quand bien même les ONG tenteraient de favoriser l'émergence d'un 
pouvoir ouvrier transcendant la particularité de chaque conflit, il est fort peu probable que les autorités acceptent l'institutionnalisation d'organisations ouvrières indépendantes des syndicats ou la démocratisation de ces derniers. Pourtant, en signant dans la pratique la faillite des syndicats officiels, en forgeant chez les ouvriers une identité commune ainsi qu'une communauté de valeurs, en leur transmettant savoir-faire, confiance et conviction d'être dans leur bon droit, cette mobilisation jette les bases sociales d'une possible remise en cause des fondements politiques du régime.

Chloé Froissart est chercheure au Centre d'études français sur la Chine contemporaine (UMIFRE $n^{\circ} 18$, Hong Kong) et directrice du Centre franco-chinois de I'Université de Tsinghua à Pékin. Ses recherches portent sur les questions de travail, de mobilisation et de développement des organisations sociales et, plus généralement, sur la citoyenneté en Chine. Elle est notamment l'auteure de La Chine et ses migrants. La conquête d'une citoyenneté (Rennes, Presses universitaires de Rennes, 2013); de "Using the Law as a "Harmonious Weapon": The Ambiguities of Legal Activism in Favour of Migrant Workers in China ", Journal of Civil Society (10 (3), 2014, p. 255-272), et de "Les "ONG" de défense des droits des travailleurs migrants : des organisations proto-syndicales qui contribuent à la stabilité du régime ", Perspectives chinoises (2, 2011, p. 20-28).

Chloefroissart@cefc.com.hk

chloefroissart@gmail.com 


\section{Critique internationale}

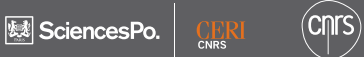

Thema

\section{Les conflits du travail dans le monde}

Volume 2. Défendre les travailleurs : acteurs, pratiques et enjeux internationaux des relations professionnelles Après une première livraison (64, juillet-septembre 2014) consacrée à la genèse des mobilisations du travail et à leurs différentes formes, ce second volet traite plus spécifiquement des organisations de défense des travailleurs. S'il est largement absent au niveau international, le syndicalisme occupe dans certains pays une place économique et institutionnelle majeure. La représentation des salariés, vecteur d'importantes dynamiques sociales et politiques, devient alors souvent un véritable enjeu national. L'étude de cas aussi variés que l'Afrique du Sud, l'Espagne, la Chine, I'Allemagne et les États-Unis permet de mettre en évidence la manière dont ces formations s'organisent et se disputent le leadership des travailleurs, et ce dans des contextes souvent défavorables. Dès lors, même si elles s'intéressent aux formes traditionnelles de régulation du travail, les cinq contributions présentées ici rappellent également le rôle central des catégories du travail dans l'intelligibilité des transformations sociales, notamment démocratiques, en offrant une autre lecture des liens entre l'économique et le politique.

\section{Varia}

\section{État prébendier et politiques industrielles au Cambodge}

par François Bafoil

\section{Affection, désaffection et défection chez deux jeunes Frères musulmans en Égypte par Marie Vannetzel}

Les « causes nationalistes » : retour sur l'adhésion militante à partir de récits biographiques

par Humberto Cucchetti

\section{État de littérature}

L'histoire des relations internationales aujourd'hui

par Pierre Grosser

\section{Derniers thema parus :}

Vers un renouveau de l'État développeur en Asie?

\section{Presses de Sciences Po}

117, boulevard Saint-Germain - 75006 Paris - France

Tél. : +33 (0)1 45498364 - Fax : +33 (0)1 45498334 - Diffusion/distribution CDE/SODIS

www.pressesdesciencespo.fr

Retrouvez la revue sur www.cairn.info et www.persee.fr

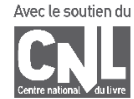

www.tentrenationalduliure.

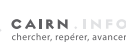

Persee
22,00€

SODIS 768311.2

ISSN 1290-7839

ISBN 978-2-7246-3351-1
SciencesPo. Les Presses

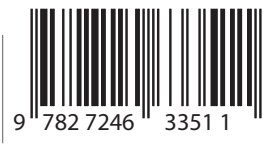

